\title{
Blutgruppenbestimmung mit synthetischen Rezeptoren - Entwicklung eines Fließsystems zur kontinuierlichen Messung
}

\author{
Prof. Franz L. Dickert, Dr. Stephan Aigner \\ Dr. Cristoph Jungbauer \\ Institut für Analytische Chemie, Universität Wien, Währinger Str. 38, A-1090 Wien \\ Österreichisches Rotes Kreuz, Wien \\ Tel.: +43-1-4277-52301, e-mail: Franz.Dickert@univie.ac.at
}

\section{Zusammenfassung:}

Im Jahr 1901 entdeckte der Serologe Karl Landsteiner das AB0-System der Blutgruppen, wodurch Bluttransfusionen ermöglicht wurden. Heutzutage werden die Blutgruppen mittels eines Antikörper-Tests bestimmt. Die Antikörper sind auf einem Dextran-Gel immobilisiert und dienen zur Detektion der Blutgruppe. Eine innovative Alternative besteht in der Anwendung von synthetischen Antikörpern, die durch Templatsynthese über das Prägen von Polymeren mit nativen Erythrozyten zugänglch sind. Bei dieser Strategie können einfache und preisgünstige Monomerbausteine verwendet werden, die zu robusten Materialien führen. Als Polymere können beispielsweise Polyvinylpyrrolidon, Polyacrylat oder Polyurethan herangezogen werden. Die Transducer werden mit diesen strukturierten Polymeren beschichtet und mit einem Fließ-System verbunden. So können mit einem reversiblen Sensorsystem Erythrozyten im Blut bezüglich des AB0 Systems, aber auch der Subgruppen typisiert werden.

Die selektive Extraktion der Erythrozyten ist z. B. über den Schwingquarz (QCM) mit der Rersonanzfrequenz von $10 \mathrm{MHz}$ möglich. Interdigitalkondensatoren (IDK) mit Strukturbreiten von $10 \mu \mathrm{m}$ bzw. $5 \mu \mathrm{m}$ ermöglichen eine einfachere Wechselstrom-Messtechnik. Die Anlagerung der Erythrozyten an die Sensorschicht führt zu einer Störung des Feldlinienverlaufs. Deshalb sind sowohl kapazitive als auch resistive Messungen möglich. Suspendiert man die Erythrozyten im entionisierten Wasser, so platzen sie aufgrund der Osmose auf, Elektrolyt wird freigesetzt und der Widerstand sinkt. Die Erythrozyten der Blutgruppen $A, B, A B$ und 0 besitzen unterschiedliche Antigene auf den Membranen und können so unterschieden werden. Auch die Subblutgruppen A - A1, A2, A1B, A2B - konnten so typisiert werden, obwohl sie sich nur aufgrund der Anzahl der Antigene auf der Zellmembran unterscheiden.

\section{Summary:}

In the year 1901 the serologist Karl Landsteiner discovered the AB0-sytem of blood-groups. In this way blood transfusion was possible. Today, blood group typing is performed by natural antibodies. The antibodies are immobilized at a dextran gel, which allows blood group typing. An innovative alternative is based on the application of synthetic antibodies, which are synthesized by imprinting of polymers with native erythrocytes. Simple, low cost monomers can be used. In this way robust materials are generated by this strategy. Polyvinylpyrrolidons, polyacrylates or polyurethanes are suitable for this purpose. The transducers are coated by patterned polymers and combined with a flow system. In this way erythrocytes in blood can be characterized according to the AB0 groups, but also in respect to sub-groups.

Selective extraction of erythrocyctes can easily be performed with e.g. quartz crystal balance working at $10 \mathrm{MHz}$. Interdigital capacitors (IDC) with structure widths of $10 \mu \mathrm{m}$ or $5 \mu \mathrm{m}$ allow the application of a more easily to be handled alternating current metrology. The adhesion of the erythrocytes will distort the electrical field lines. Thus, both capacitive and resistive measurements are possible. If the erythrocytes are suspended in deionized water, these cells will be destroyed due to osmotic effects resulting in a resistance decrease. The erythrocytes of blood groups $A, B, A B$ und 0 are characterized by different antigens in the membranes which will lead to a biological recognition process. Even a sub group typing $A$ $-A_{1}, A_{2}, A_{1} B, A_{2} B$ - can be performed, in spite of the fact that only the amount of antigens in the cell membrane are different.

\section{Einleitung:}

Die Blutgruppenserologie beschäftigt sich mit der Reaktion von Antigenen mit Antikörpern im Humanblut. Bis heute sind etwa 550 Antigene entdeckt worden, es ist aber nur ein geringer Anteil für die Transfusionsmedizin von Bedeutung [1]. In der Transfusionsmedizin wird üblicherweise das AB0-System verwendet. Dabei werden die Antigene in A-Antigene, B-Antigene und $\mathrm{H}$-Antigene eingeteilt. Die Erythrozyten unterscheiden sich durch Antigene auf der Membran der Blutkörperchen, die einen ungefähren Durchmesser von 7-8 $\mu \mathrm{m}$ aufweisen (Abb. 1). Diese Zellen sind sehr flexibel, damit der Sauerstofftransport auch in Kapillargefäßen gewährleistet ist. Eine Unterscheidung der Blutgruppen erfolgt über die Antigene auf der Zellmembran, A-Antigene, B-Antigene und H-Antigene (Abb. 2). Das H-Antigen ist hierbei das Grundsystem, das sich aus der Vorläufersubstanz mittels der a-L-Fucosyl-Transferase bildet. An die Galaktose-Gruppe der Zellmembran wird eine Fucose-Gruppe gebunden. Die A-Antigene werden 


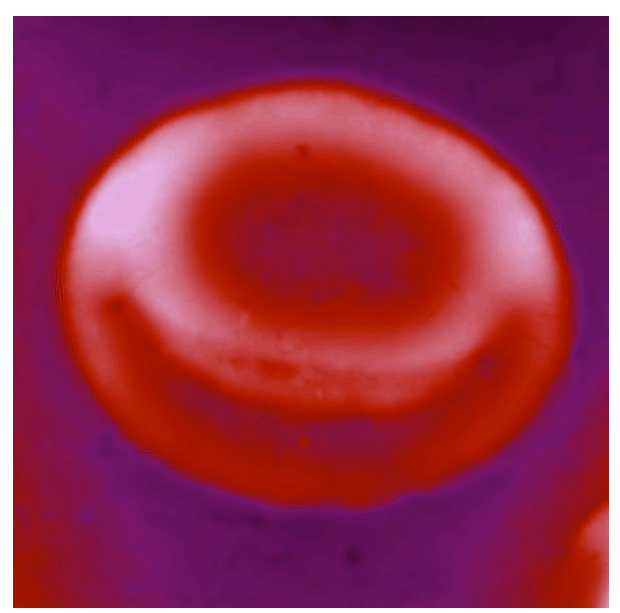

Abb. 1: AFM Bild eines Erythrozyten über das $\mathrm{H}$-System mittels der $\alpha-\mathrm{N}$-AcetylgalaktosaminTransferase gemäß der N-Acetylgalaktosamin-Gruppe gebildet. Für die Herstellung der B-Antigen-Struktur wird mittels $\alpha$-Galaktosyl-Transferase eine weitere Galaktose an das $\mathrm{H}$-System angehängt und so entsteht die Blutgruppe $\mathrm{B}$. Diese Schritte sind in Abb. 2 schematisch dargestellt. Sind sowohl A- als auch B-Antigene auf den Erythrozyten vorhanden, spricht man von der Blutgruppe $A B$. Blutgruppe 0 liegt vor, wenn an der $\mathrm{H}$-Substanz keine weiteren Zucker gebunden sind. Damit differenzieren sich die AB0Determinanten nur durch die unterschiedlichen terminalen Zuckermoleküle.

Weiterhin kann man im AB0-System zusätzliche Subgruppen klassifizieren, die sich über unterschiedliche Antigen-Antikörper Wechselwirkungen erkennen lassen. So unterscheiden sich die A-Subblutgruppen $A_{1}$ und $A_{2}$ durch die unterschiedliche Anzahl von Antigenen auf der Erythrozytoberfläche. Die Subblutgruppe $A_{1}$ besitzt eine etwa zwei- bis dreifach höhere A-Antigenkonzentration auf einer Blutzelle als die Subgruppe $A_{2}$. Auch die beiden Subgruppen $A_{1} B$ und $A_{2} B$ unterscheiden sich durch die unterschiedliche Zahl der A-Antigene. Die
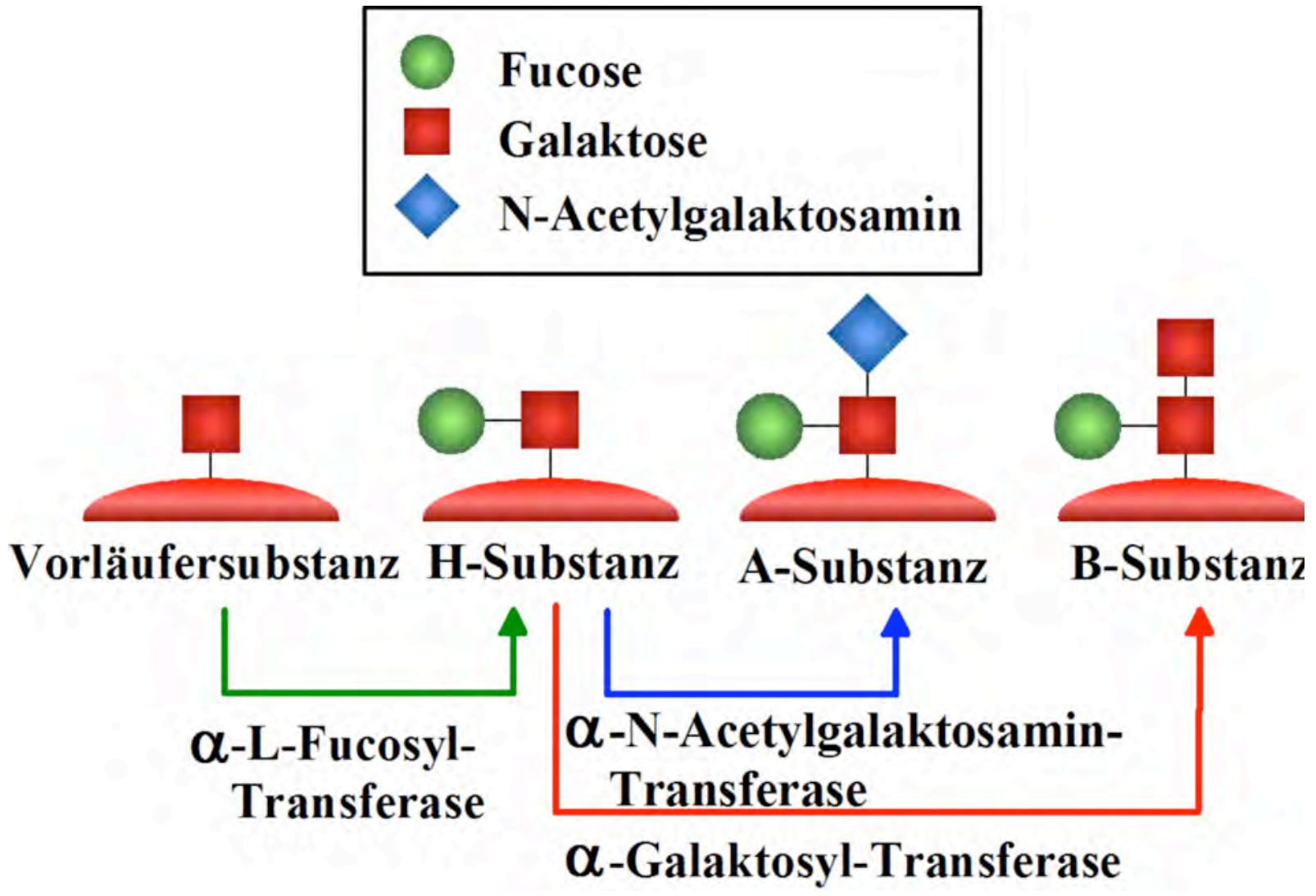

Abb. 2: Antigene des ABO Blutgruppensystems

Subblutgruppen $A_{1}$ und $A_{2}$ stellen jedoch für die Transfusionsmedizin keine relevante Gefahr dar, da die Antigenanzahl auf den Blutzellen kein transfusionsmedizinisches Problem darstellt. Die Blutgruppe 0, die nur $\mathrm{H}$-Antigene besitzt, ist somit Universalspender und die Blutgruppe AB fungiert aufgrund der A- und BAntigene als Universalempfänger. Bei der Verabreichung einer falschen Blutgruppe kommt es zur Agglutination der Blutkörperchen. Weiterhin ist bei der Transfusionsmedizin der Rhesusfaktor von Bedeutung [2]. Dieser wurde von Karl Landsteiner und Alexander Solomon Wiener 1940 entdeckt und wird heutzutage meistens mit der AB0-Blutgruppe zusammen bestimmt und angegeben. Der Rhesusfaktor ist ein Protein auf der Zellmembran der Erythrozyten und besteht aus 417 Aminosäuren und beeinflusst im Körper den Ammonium-lonen- und Ammoniak-Transport. 
Das menschliche Blut liegt als Suspension von Blutplasma und zellulären Bestandteilen vor. Dabei macht das Blutplasma $55 \%$ und die Zellen $45 \%$ bezüglich des Volumens aus. Im Plasma sind gelöste Salze und niedermolekulare Verbindungen enthalten. Die Konzentration der roten Blutkörperchen im Blut beträgt zwischen 4,0 und 6,5 Millionen pro $\mathrm{mm}^{3}$ und so ergibt sich im Körper eine Gesamtanzahl von ca. 20 bis 30 Billionen. Die Erythrozyten haben eine durchschnittliche Lebensdauer von 130 Tagen. In der Membran sind eine hohe Anzahl von Proteinen integriert, die für den Transport von Elektrolyten verantwortlich sind. Dieser Austausch von lonen ist für die bikonkave Form der Zelle notwendig und über die Osmose erfolgt ein Angleichen an die Umgebung. Nur bei einer isotonischen Umgebungsbedingung (154 mmol// NaCl) ist die Stabilität der Zelle gewährleistet, ansonsten kommt es zu einer Schrumpfung oder Platzen der roten Blutkörperchen.

\section{Ergebnisse und Diskussion:}

Ila. Sensitive Schichten - Molekulares Prägen Generell muss zum Nachweis eines Analyten eine chemische Erkennung durchgeführt werden. Hierzu entwirft man sinnvollerweise eine Sensorschicht, die eine selektive Anreicherung des Analyten ermöglicht. Diese Strategie kann durch Biorekognition realisiert werden. Ein Grundphänomen hierbei sind üblicherweise sterische Beiträge, wodurch die Geometrie eines Moleküls charakterisiert wird. Dieser geometrische Effekt ist aber mehr ein Ausschlussprinzip, ein Partikel, das nicht in eine Kavität hineinpasst wird auch nicht eingelagert. Zur Bindung des Analyten an eine Schicht ist aber der enthalpische Beitrag von entscheidender Bedeutung, damit die Gibbs Energie ein negatives Vorzeichen erhält. Diese enthalpische Wechselwirkungen zwischen einem Erythrozyten und der Sensoroberfläche ist besonders wichtig, da die roten Blutkörperchen eine flexible Gestalt aufweisen. So ist die Geometrie bei der Anreicherung der Erythrozyten an die Sensorschichten nicht ausschlagend, da diese Zellen eine flexible Form aufweisen. Die enthalpischen Beiträge resultieren auf intermolekularen, nichtkovalenten Wechselwirkungen zwischen den Antigenen des AB0-Systems und den Rezeptoren auf der Sensorschicht. Zur Erken-
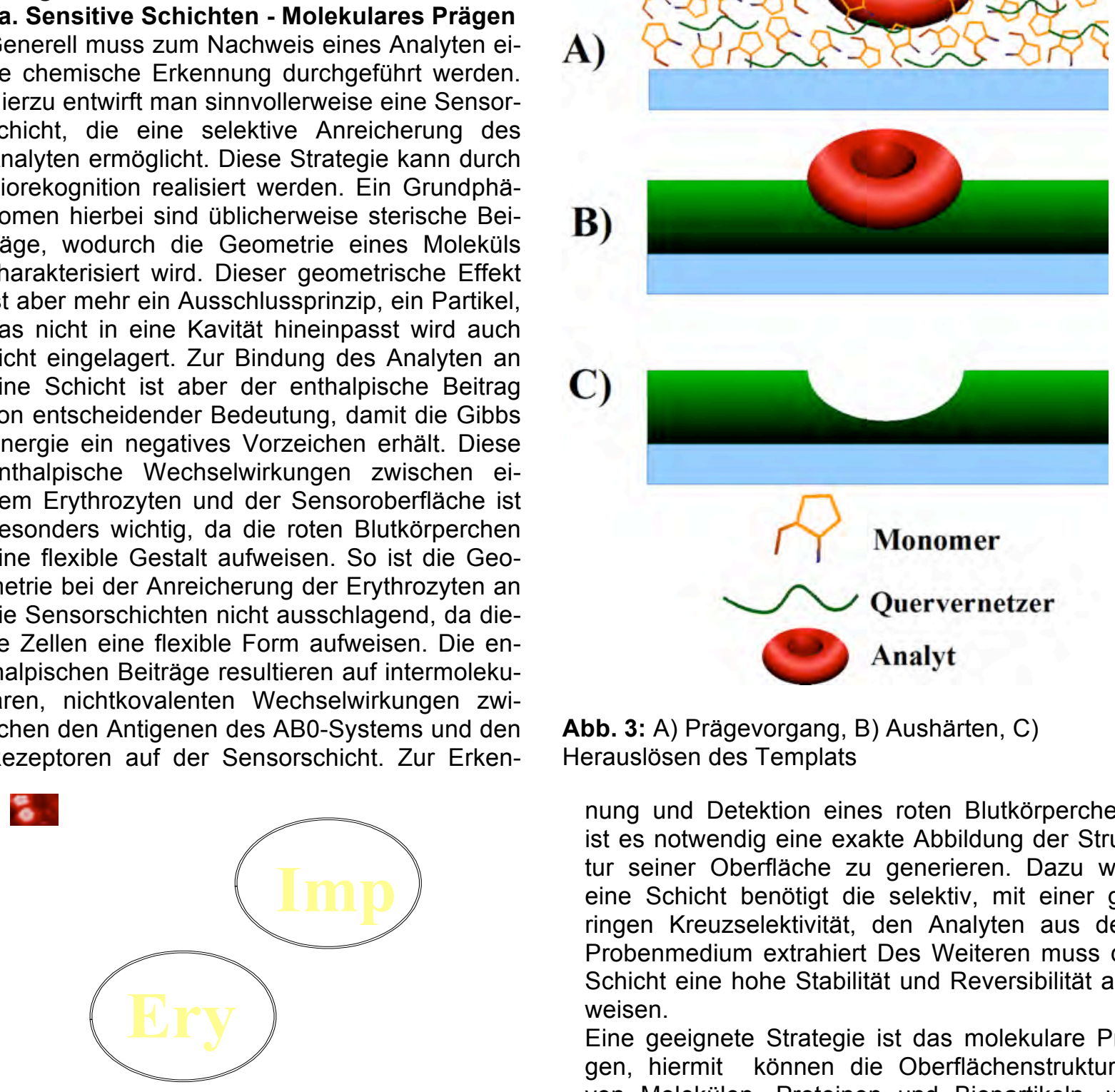

Abb. 3: A) Prägevorgang, B) Aushärten, C) Herauslösen des Templats

nung und Detektion eines roten Blutkörperchens ist es notwendig eine exakte Abbildung der Struktur seiner Oberfläche zu generieren. Dazu wird eine Schicht benötigt die selektiv, mit einer geringen Kreuzselektivität, den Analyten aus dem Probenmedium extrahiert Des Weiteren muss die Schicht eine hohe Stabilität und Reversibilität aufweisen.

Eine geeignete Strategie ist das molekulare Prägen, hiermit können die Oberflächenstrukturen von Molekülen, Proteinen und Biopartikeln, wie Zellen und Viren, auf Polymerschichten abgebildet werden [3]. Diese Materialien können als synthetische Antikörper betrachtet werden und sind im Gegensatz zu den natürlichen Analoga gegen Degradationsvorgänge robuster. Die räumliche Strukoberfläche - Erythrozyt und Imprint

tur der Rezeptorstellen wird durch eine hohe Quervernetzung des Polymers erreicht. Zur Herstellung dieser strukturierten Polymere drückt man die Erythrozyten auf einem planaren Substrat in ein Vorpolymer. Dabei orientieren sich die noch verbliebenen Monomere und Oligomere zu den Wechselwirkungszentren der Zelloberfläche. Nach dem Aushärten entsteht ein rigides Polymer. Das Templat kann ausgewaschen werden und die strukturierten Oberflächen binden dann wiederholt Erythrozytzellen. Dieses 
Prägeverfahren gemäß einer Templatstrategie (Abb. 3) kann dann bei den unterschiedlichsten Transducern angewendet werden. Besonders geeignet sind Polyurethane oder Polyvinylpyrrolidone, die durch bifunktionelle Monomere vernetzt sind. In jedem Fall spielen bei diesen beiden Polymeren die Ausbildung von Wasserstoffbrückenbindungen zu den Antigenen des AB0-Systems eine besondere Bedeutung. Das Herauslösen und die Wiedereinlagerung der Erythrozyten kann direkt mit AFM beobachtet werden, das eingelagerte rote Blutkörperchen und das Imprint (Kavität) können deutlich, wie in Abb. 4 gezeigt, visualisiert werden.

\section{IIb. QCM-Messungen:}

Die selektive Extraktion der unterschiedlichen Erythrozyten aus wässriger Lösung kann mit massensensitiven Transducern, insbesondere dem Schwingquarz (QCM) als universelle Detektionsmethode durchgeführt werden. Da die durchschnittlichen Abmessungen der Blutkörperchen bekannt sind, kann nach der Sauerbrey Gleichung über die Frequenzantwort unmittelbar die Zahl der adsorbierten Partikel bestimmt werden, die ein Maß für die Konzentra-

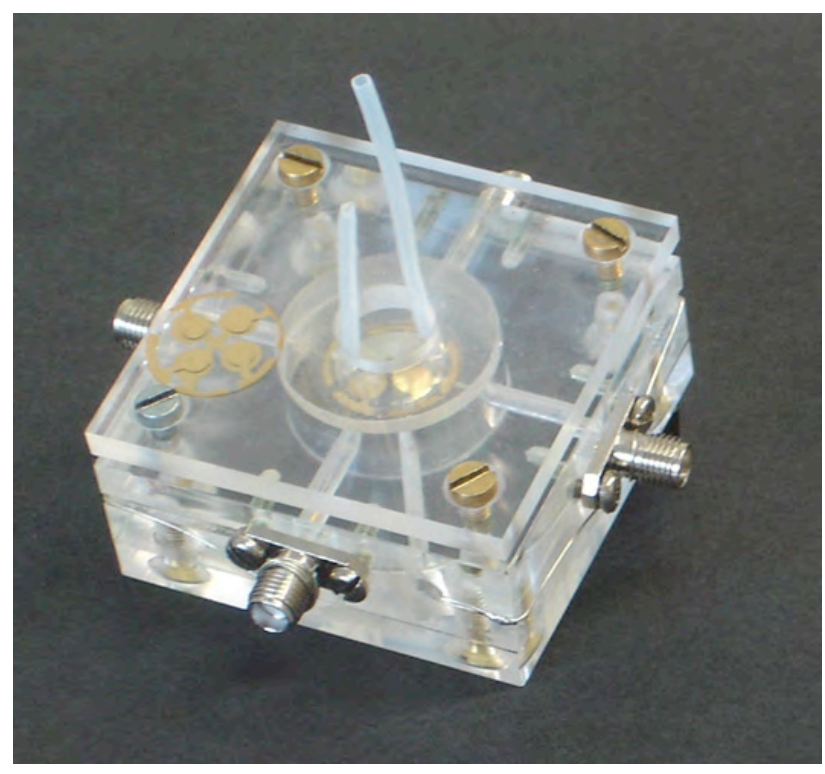

Abb. 5: QCM-Mess-Zelle mit Quarzen tion in Lösung darstellt. Die $10 \mathrm{MHz}$ QuarzBlanks im Durchmesser von 14,5 mm wurden jeweils im asiatischen Raum gekauft und dann zwei bis vier Elektroden mit Siebdruck aufgebracht. Die mit Goldpaste strukturierten Quarze waren nach dem Einbrennvorgang bei ca. $400^{\circ} \mathrm{C}$ und einem Waschprozess mit Methylpyrrolidon verwendungsfähig. Die fertigen Quarze werden in einer Durchfluss-Zelle mit Elektroden zur Durchführung der Messungen kontak-

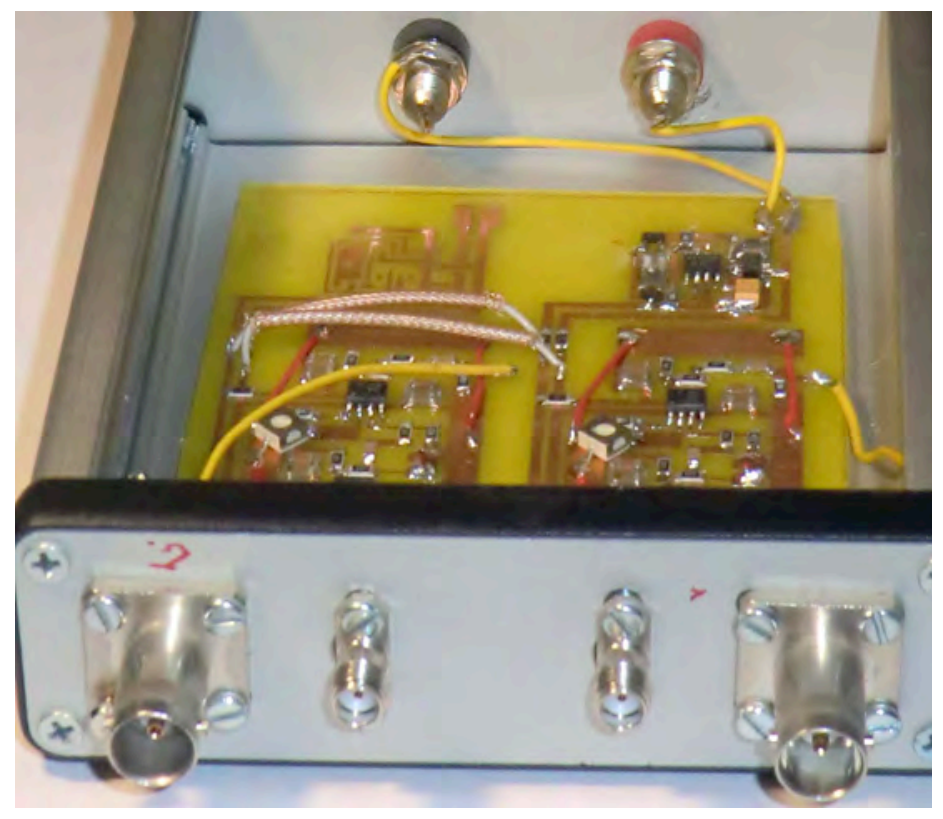

Abb. 6: QCM - Oszillator tiert (Abb. 5). Es werden jeweils mindestens zwei Elektrodenanordnungen eingesetzt, da so z. B. Temperaturfluktuationen kompensiert werden können. Die massensensitiven QCM-Messungen lassen sich mit einer in der Arbeitsgruppe erstellten Oszillatorschaltung betreiben. Hierzu werden zweistufige Verstärker mit einer Oszillator- und einer Ausgangsstufe mit Operationsverstärkern der Grenzfrequenz 600-900 MHz herangezogen (Abb. 6).

Alle Elektroden der $10 \mathrm{MHz}$ Quarze wurden mit einem vernetzten Polyvinylpyrrolidon beschichtet [4]. Die Prägung erfolgte mit den jeweiligen Erythrozyten geeigneter Konzentration (z. B. c = $1,0 * 10^{7}$ Zellen/ $\left.\mu \mathrm{l}\right)$. Eine Elektrode wurde zwar auch mit Polyvinylpyrrolidon beschichtet, allerdings nicht geprägt. So können alle Hintergrundeffekte einschließlich Temperatureinflüsse größtenteils kompensiert werden. Die Messlösungen wurden aus den verschiedenen Blutgruppenproben hergestellt und jeweils eine verdünnte Lösung in destilliertem Wasser der Messzelle zugeführt. Die Pumpgeschwindigkeit beim Befüllen, Entleeren und Waschen der Messzelle betrug ca. 0,5 ml/min. Die Peristaltikpumpe saugt dabei die Erythrocyten durch die Zellen, damit sie nicht beim Pumpvorgang zerquetscht werden. 
Wird ein Quarz mit einer $A_{1}$-geprägten Elektrode in die Messzelle eingebaut, so erhält man die signifikanteste Frequenzänderung, wenn Templat und Analyt identisch sind (Abb. 6). Dies ist darauf

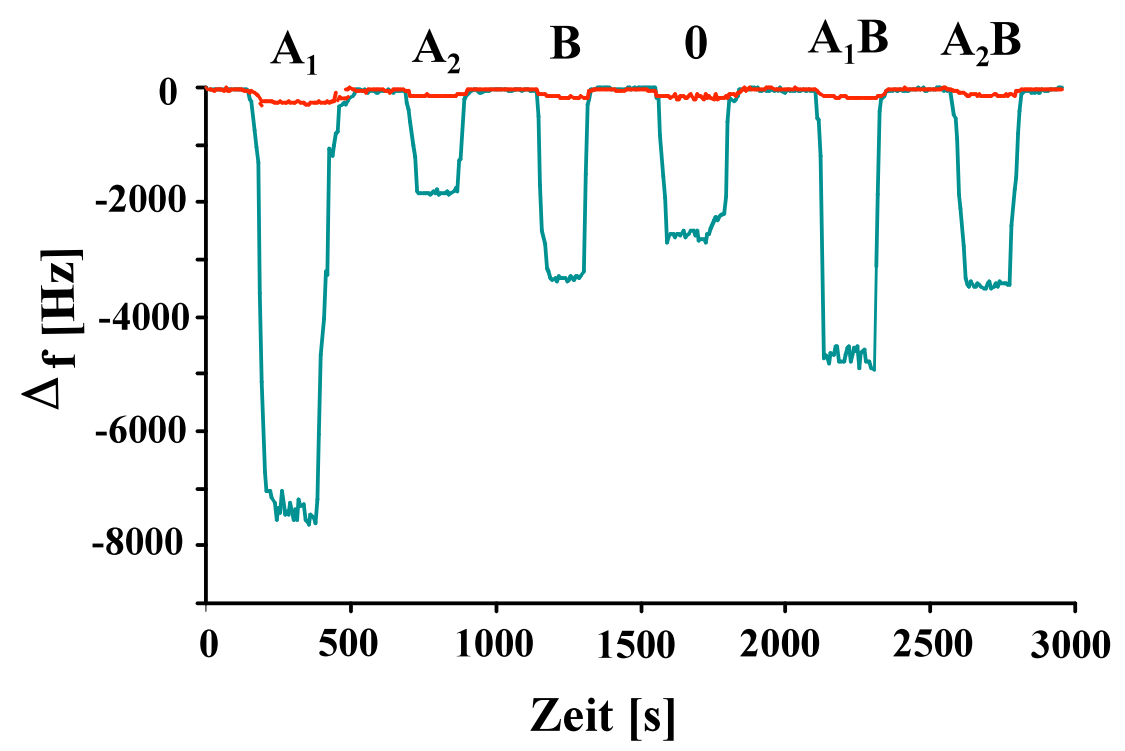

Abb. 6: $10 \mathrm{MHz}$ Sensor-Antwort, die sensitive Schicht ist mit der Blutgruppe $A_{1}$ geprägt

zurückzuführen, dass die Erythrozyten der $\mathrm{A}_{1}$ Blutgruppe am stärksten von den Kavitäten dieser Polymerschicht gebunden werden. Offenbar verhält sich das adsorbierte Blutkörperchen ähnlich wie die sensitive Schicht als rigider Festkörper und es resultiert eine Frequenzerniedrigung von ca. $7500 \mathrm{~Hz}$, was sich über die Sauerbrey Gleichung erklären lässt. Die Referenzelektrode zeigt nur eine geringe Frequenzantwort, was die Bedeutung einer erfolgreichen Prägung unterstreicht, Dieses Polymer liefert somit nur sehr geringe unselektive Hintergrundeffekte von ca. $240 \mathrm{~Hz}$, also einen um den Faktor 30 geringeren Wert als die Antwort der Messelektrode auf den Analyten.

Es wird also bei einer $A_{1}$ - Prägung wie in Abb. 6 gezeigt beim Analyten $A_{1}$ das optimale Ergebnis

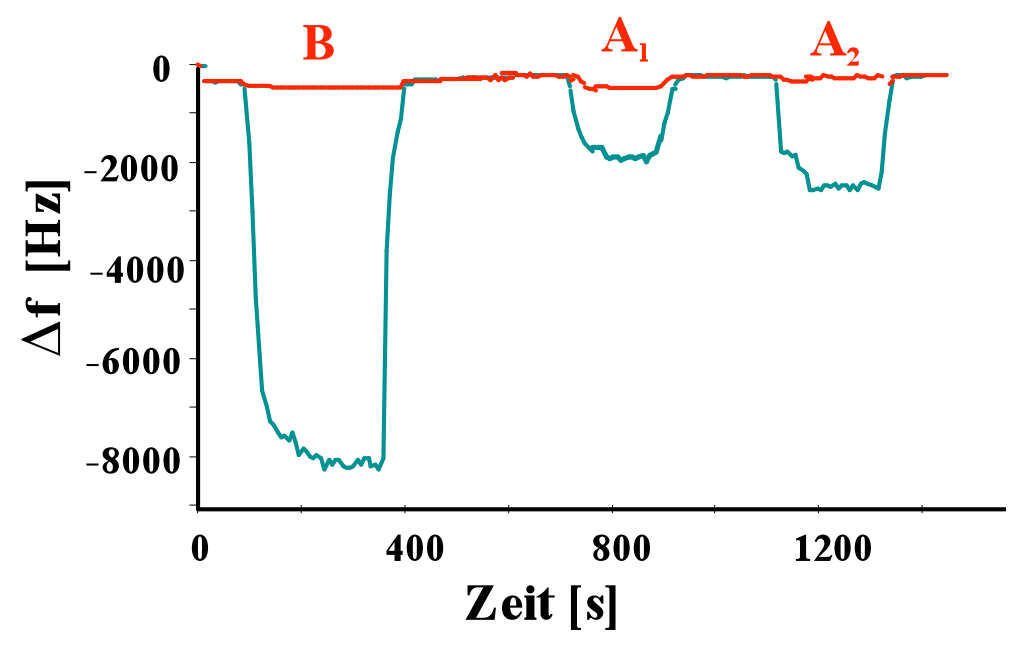

Abb. 7: $10 \mathrm{MHz}$ Sensor-Antwort, sensitive Schicht geprägt mit der Blutgruppe B membran optimale Wechselwirkungen. Die Erythrozyten der Blutgruppe $A_{1} B$ (420000-850000 A-Antigene bzw. 310000-560000 B-Antigene), liefert so neben der Templatblutgruppe die zweitgrößte Wechselwirkung. Im Gegensatz dazu wird die Blutgruppe $A_{2}$ mit ihren 160000 bis 440000 A-Antigenen und H-Aktivität nicht so effektiv gebunden, was sich unmittelbar in der Frequenzänderung niederschlägt. Wird $z$. B. die Polymerschicht auf der Messelektrode mit der Blutgruppe $A_{2}$ geprägt, so zeigt das Templat wiederum die höchste Frequenzerniedrigung von $8150 \mathrm{~Hz}$. Die Blutgruppe $\mathrm{A}_{2} \mathrm{~B}$, die $120000 \mathrm{~A}$-Antigene und 310000 
bis 560000 B-Antigene aufweist, liefert eine Änderung von $5750 \mathrm{~Hz}$. Die anderen vier untersuchten Blutgruppen zeigen bei diesem Sensor nur eine geringe Änderung im Bereich von 2500 bis $1300 \mathrm{~Hz}$.

Eine sehr gute Selektivität liefert auch ein mit der Blutgruppe B geprägter Sensor. Wie die Messkurve in Abb. 7 zeigt ergibt das Templat wiederum das höchste Signal, während alle anderen Blutgruppen wesentlich kleinere Veränderungen bezüglich der Frequenzen liefern. Durch die hohe Anzahl an B-Antigene auf der Zellemembran der B-Erythrozyten (610000-830000) können die anderen Blutzellen nicht so gut von den geprägten Kavitäten inkludiert werden. So liefert die Blutgruppe $B$ eine Frequenzänderung von $7880 \mathrm{~Hz}$ während die Blutgruppe $A_{2}$ eine Antwort von $2230 \mathrm{~Hz}$ und die Blutgruppe $A_{1}$ mit $1750 \mathrm{~Hz}$ einen ebenfalls geringen Effekt hervorruft.

Im Vergleich zu der vorangegangenen Messung mit einem Blutgruppen B-Sensor zeigt ein mit der Blutgruppe 0 geprägter Sensor ein völlig unterschiedliches Muster, wie in Abb. 8 gezeigt. Das Templat mit der Blutgruppe 0 liefert dabei eine Frequenzerniedrigung von $5410 \mathrm{~Hz}$, während die Blutgruppen $A_{1}, A_{2}$ und $B$ sich nur sehr unwesentlich bezüglich der Sensorantwort davon unterscheiden. Bei nä-

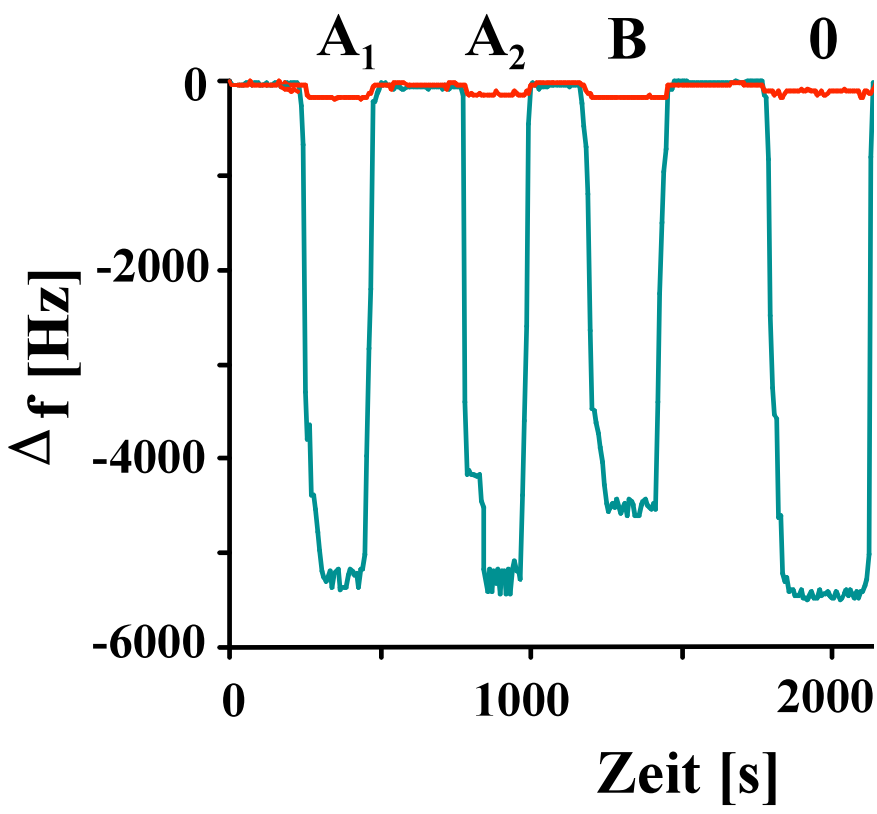

Abb. 8: $10 \mathrm{MHz}$ Sensor-Antwort, sensitive Schicht geprägt mit der Blutgruppe $A_{1}$ herer Betrachtung ist dies nicht verwunderlich, da die Blutgruppe 0 nur $\mathrm{H}$-Antigene auf der Oberfläche aufweist und diese als Ausgangsverbindung für die anderen Blutgruppen dienen. Die beträchtlichen Unterschiede zwischen den Sensorantworten bei der Prägung mit A / B Erythrocyten basieren auf den Zuckern Galaktose und N-Acetylgalaktosamin. Dies zeigt die beträchtlichen Potentiale des molekularen Prägens mit Zellen, dass die Variation nur eines Zuckers die Ergebnisse so entscheidend verändert (Abb. $6,7)$.

\section{Ilc. Kapazitive Messungen:}

Für die Differenzierung der Blutgruppen lassen sich auch Interdigitalkondensatoren (IDK) mit einer Polyvinylpyrrolidon-Schicht, die mit der jeweiligen Blutgruppe geprägt ist, verwenden. Zur Detektion kann dann die Kapazität herangezogen werden. Die Durchführung dieser Messmethode ist noch einfacher und preisgünstiger als mit massensensitiven Hochfrequenzbauteilen zu handhaben. Die herangezogenen Interdigitalkondensatoren haben vorzugsweise einen Elektrodenabstand und Elektrodenbreite von $10 \mu \mathrm{m}$, bzw. $5 \mu \mathrm{m}$. Es sind besonders signifikante Effekte zu erwarten, wenn die Erythrozyten ähnliche Durchmesser wie die Dimension der IDK Strukturbreiten aufweisen. Die Blutproben wurden vor der Messung frisch hergestellt, und spätestens 5 Minuten nach deren Mischung gemessen. Dadurch soll das Platzen beziehungsweise Verklumpen der Blutzellen vermieden werden. Wie-

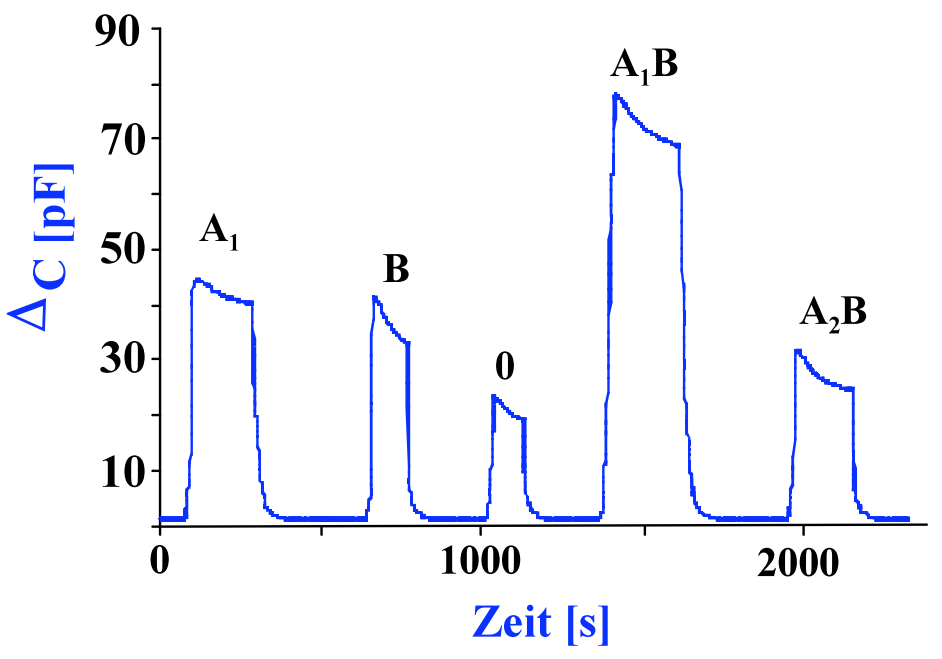

Abb. 9: Kapazitiver AB0 - Sensor, Polyvinylpyrrolidon, geprägt mit $A_{1} B$ derum wird eine Peristaltikpumpe eingesetzt.

Bei der Messung mit einem $A_{1} B$-geprägten Sensor weist die Templatblutgruppe mit einer Kapazitätsänderung von $55 \mathrm{pF}$ den größten Effekt auf. Aufgrund der Antigenstrukturen auf der Erythrozyten- 
membran, die 420000 bis 850000 A-Antigene und 310000 bis 560000 B-Antigene besitzt, zeigen die Blutgruppen $A_{1}$ (810000-1700000 A-Antigene) und B (610000-830000 B-Antigene) die höchste Querempfindlichkeit auf. Dabei nimmt die Kapazität bei Messung der $A_{1}$-Erythrozyten um $40 \mathrm{pF}$ und der Blutgruppe B um 33 pF zu. Die Blutgruppe $A_{2} B$ mit 120000 A-Antigene und 310000 bis $560000 \mathrm{~B}-$ Antigene liefert eine Kapazität von $25 \mathrm{pF}$. Die Blutgruppe 0, die nur H-Antigene auf der Zellmembran aufweist, stellt dabei die Blutgruppe mit dem geringsten Signal bei Verwendung eines $A_{1} B$-Sensors dar (Kapazität 19 pF). Aufgrund unterschiedlicher Kapazitätsmessungen mit dem IDK-Sensor sind die sechs Blutgruppen des AB0-Systems voneinander zu unterscheiden. Die Änderung der Kapazität ist immer für die Templatblutgruppe im ABO - System am größten.

\section{Ild. Resistive Messungen:}

Das grundlegende Phänomen beim Nachweis von Erythrozyten ist die Biorekognition durch eine sensitive Schicht. Dies gilt auch für den resistiven Nachweis. Sehr wesentliche Aspekte sind hierfür osmotische Effekte und damit auch verbunden die lonenkonzentration in Lösung. Verwendet man eine isotonische $\mathrm{NaCl}$ Lösung so wird, wie Abb. 10a dargestellt, die Form und Größe des roten Blutkörperchen erhalten. Das leitende Medium des $\mathrm{NaCl}$ in Wasser führt zu einer beträchtlichen Leitfähigkeit, da

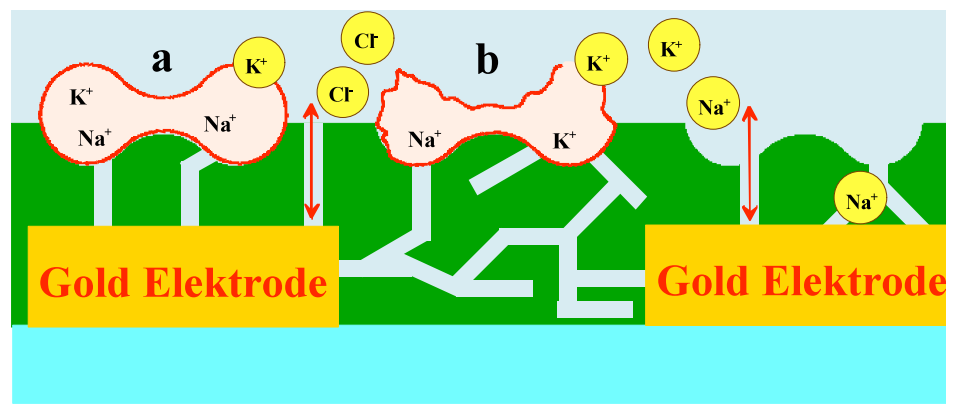

Abb. 10: Beschichteter Interdigitalkondensator, Adsorption von Erythrozyten die lonen einen geringen Widerstand zwischen den Elektroden verursachen. Die Polymere, wie z. B. quervernetzte Polyvinylpyyrolidone, sind

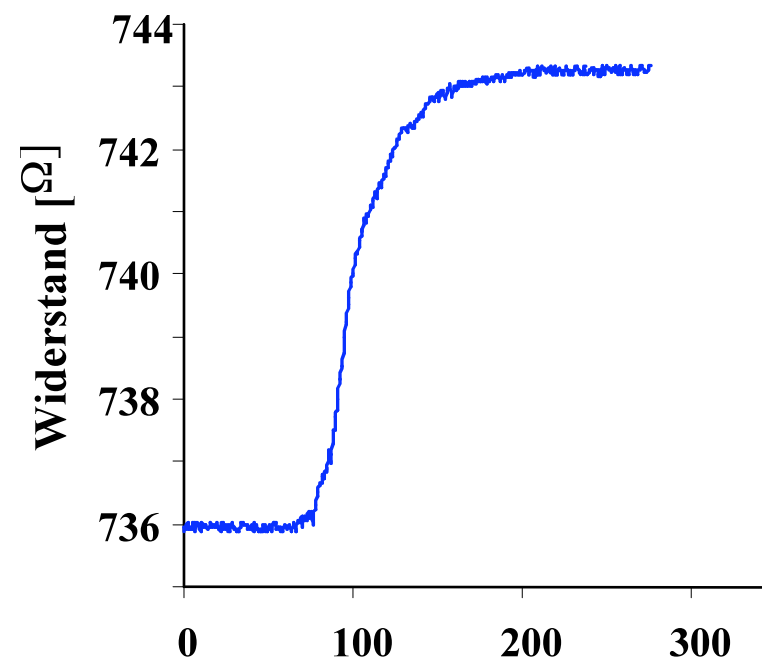

Abb. 11: Adsorption eines Erythrozyten auf einem polymerbeschichteten $10 \mu \mathrm{m}$ IDK in isotonischer Lösung nach der hier verwandten Darstellungsmethode porös und weisen Diffusionskanäle auf. Zudem zeigen diese Materialien eine hohe Polarität und so findet vor allem über Wasserstoffbrückenbindungen eine ausgeprägte Absorption von Wassermolekülen statt. In diesem Medium mit hoher Dielektrizitätskonstanten bilden sich freie lonen, die nicht assoziiert sind und es resultiert eine hohe Leitfähigkeit. Das Polymer ist mit dem Templat $A_{1}$ geprägt und so sind optimale Wechselwirkungszentren zur identischen Blutgruppe generiert. Die Adsorption der Blutkörperchen führt nach Abb. 11 über eine Wechselstrommessung im $\mathrm{kHz}$ Bereich zu einer Widerstandserhöhung. Offenbar erfolgt nach Abb. 10a eine partielle Blockierung der Sensoroberfläche, wodurch ein Teil der Ionenkanäle keinen Leitfähigkeitsbeitrag liefern können.

Eine andere Messmethode kann nach Abb. 10b herangezogen werden. Der Sensor wird vor der ersten Anwendung intensiv mit entionisiertem Wasser gespült, um das Templat und lonen vollständig aus dem Polymer zu entfernen. Für die Messungen wurden die zu untersuchenden Blutgruppen destilliertem Wasser suspendiert. Die Blutproben wurden vor der Messung frisch hergestellt und zum Befüllen und Entleeren der Messzelle diente wiederum eine Peristaltikpumpe. Erreicht die Probenlösung die Messzelle und somit den IDK, erfolgt ein sehr schneller Abfall des Widerstandes, es stellt sich rasch ein annähernd gleichbleibender Widerstandswert ein. Die Änderung des Widerstandes hat mehrere Ursachen, die Blutzelle, die Polymerstruktur und das Medium. So besitzt das Polymer neben den Kavitäten für die Einlagerung der Blutkörperchen poröse Strukturen. Diese ermöglichen die Diffusion von geladenen Teilchen zwischen den Elektroden (Abb. 10b). Das Ausmaß der Leitfähigkeitserhöhung ist abhängig von den in Lösung befindlichen lonen und den Kavitäten der Polymerschicht. Wie in Abb. 10b dargestellt, degradieren die roten Blutzellen in hypotonischer Lösung, wodurch lonen aus dem Inneren der Zelle freigesetzt werden. Passen die Erythrozyten optimal in die 
geprägten Kavitäten so werden besonders viele lonen gebildet, d. h. es wird ein besonders niedriger Widerstandswert beobachtet.

Abb. 12 zeigt einen $A_{1}$-geprägter Sensor, der resistive Anworten auf das ABO System liefert. Bei dieser

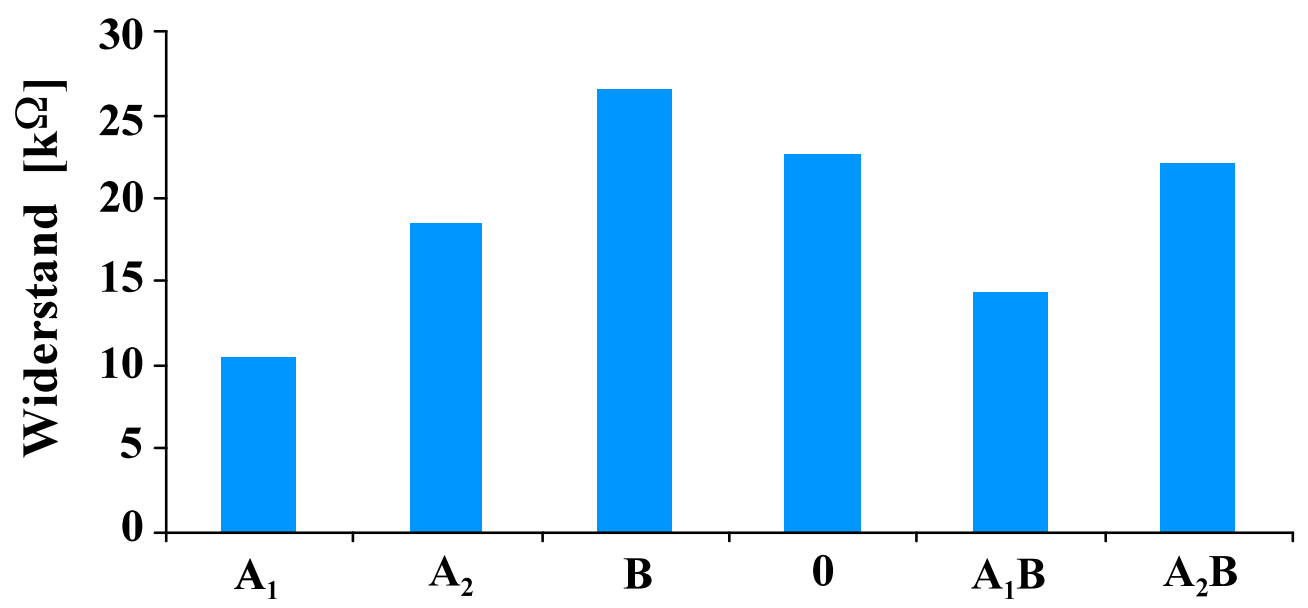

Abb. 12: Resistiver $A B 0$ - Sensor, Vinylpyrrolidon, geprägt mit $A_{1}$

Art von Messung erhält man einen minimalen Widerstandwert, wenn Analyt und Templat identisch sind. Das Muster an Antworten ist wie bei den QCM-Messungen zu verstehen. Die Zahl und Art der Antigene der Erythrozytenzellmembran steuern das Ausmaß der Adsorption, was wiederum die Zahl der austretenden lonen bedingt.

Bei diesen Widerstandsmessungen bedingen die Blutgruppen, die zur Prägung der Polymerschicht herangezogen wurden, die niedrigsten Werte. Auch die Sub-Blutgruppen der Blutgruppe A können durch die molekular geprägten Schichten mittels Messung des Widerstandes unter der Verwendung von IDKSensoren unterschieden werden. Das Sensorsystem zeigt gute Reversibilität und reproduzierbare Messwerte. Durch Spülen der Messzelle können die Anfangswerte wieder erreicht werden und eine neue Messserie lässt sich starten.

\section{Schlussfolgerungen:}

Die Typisierung von Erythrozyten nach dem AB0 - System kann durch die Kombination von molekular geprägten Poymeren mit Quarzmikrowaagen, sowie kapazitiven und resistiven Messungen mit Interdigitalstrukturen erzielt werden. Mit allen Mess-Methoden lassen sich über ein Muster von Sensorantworten auch Subgruppen erkennen. Ein Array der diskutierten Transducer liefert eine eindeutige Zuordnung der Blutgruppe. Das signifikanteste Signal wird beobachtet, wenn die prägene Blutgruppe (Templat) identisch zum Analyten ist. Sensoren bestehend aus beispielsweise strukturierten Polyvinylpyrrolidonschichten in Kombination mit den jeweiligen Transducern haben durchgehende Betriebszeiten von vielen hundert Stunden. Die erhaltenen Muster konnten auch nach langer Zeit die Blutgruppen charakterisieren. Selbst Alterungsphänomene der Blutkonserven, die aufgrund von Agglutinationsvorgängen auftreten, waren zugänglich. Dabei war zu beobachten, dass Blutproben mit einem Alter von über 3 Monaten deutliche Degradationsphänomene zeigen.

\section{Literatur:}

[1] H. Schenkel-Brunner; Human Blood Groups - Chemical and Biochemical Basis of Antigen Specificity, Springer, Wien, New York, 2000.

[2] J.P. Cartron; Defining the Rh blood group antigens - Biochemistry and molecular genetics, Blood Rev. 8, 199-212 (1994).

[3] O. Hayden, K.-J. Mann, S. Krassnig, and F.L. Dickert; Biomimetic ABO Blood-Group Typing, Angew. Chem. Int. Ed. 45, 2626 (2006).

[4] A. Seifner, P.A. Lieberzeit, C. Jungbauer, F.L. Dickert; Synthetic receptors for selectively detecting erythrocyte ABO subgroups, Analytica Chimica Acta 651, 215-219 (2009). 